\title{
Especies forestales promisorias para la diversificación de forestaciones en la Patagonia Argentina
}

\author{
Promissory forest species for the diversification of afforestations \\ in Argentine Patagonia
}

\author{
María Marcela Godoy ${ }^{a}$ b*, Guillermo E. Defosséa, b y Martin Threnc \\ *Autor de correspondencia: aCentro de Investigación y Extensión Forestal Andino-Patagónico (CIEFAP-CONICET), \\ Ruta 259 km 4, Esquel, Chubut, Argentina, C.C. 14 (9200), Tel./fax: 54- 2945-453948, mmgodoy@ ciefap.org.ar. \\ bUniversidad Nacional de la Patagonia San Juan Bosco, Facultad de Ingeniería, \\ Cátedra de Ecología Forestal, Esquel, Argentina. \\ 'Universidad de Ciencias y Artes Aplicadas, Facultad de Manejo de Recursos, Göttingen, Alemania.
}

\begin{abstract}
SUMMARY
In the western region of Patagonia, Argentina, there are about 70 thousand ha of afforestations, with $80 \%$ corresponding to Pinus ponderosa and the rest to Douglas fir (Pseudotsuga menziesii) and lodgepole pine (Pinus contorta). To avoid the tendency to this kind of monocultures, with their unfavorable ecological and sanitary consequences, there is a need of evaluating the possibility of cultivating other timber species, to allow for the diversification of the current afforestations. In this study, small stands and groups of trees planted in the last 50 years were sampled, identifying the species present and measuring height, age and diameter of trees, as well as recording the environmental conditions where they grow. Those healthy species, that were found in two or more sites, and whose growth parameters resulted higher than those found in their places of origin, were selected as promissory species. Measured dominant heights were compared with those appearing in yield tables and site index curves for their natural distribution areas, so as to evaluate their growth potential. The species that showed higher heights than the same species grown in their native areas, were healthier, had high wood quality and a recognised value in the wood market were: Quercus robur, Pinus monticola, Pinus sylvestris and Larix decidua. All demonstrated a high potential to make provenances and plantation trials to diversify afforestations in Patagonia.
\end{abstract}

Key words: forest species introduction, site index, Patagonia.

\section{RESUMEN}

En el oeste de la Patagonia argentina existen aproximadamente 70 mil hectáreas forestadas, de las cuales el $80 \%$ corresponde a Pinus ponderosa y el resto a Pseudotsuga menziesii y Pinus contorta. Con el objetivo de evitar esta tendencia hacia los monocultivos con sus consecuencias ecológicas y sanitarias desfavorables, surge la necesidad de evaluar la posibilidad de cultivar otras especies forestales que permitan la diversificación de las actuales forestaciones. En este trabajo se analizaron pequeños rodales y grupos de árboles plantados en los últimos 50 años en la región, identificando las especies presentes y midiendo en ellas las variables dasométricas, de sanidad y forma, así como las condiciones ambientales en las que crecieron. Aquellas especies sanas, que se presentaron en dos o más sitios y cuyos parámetros de crecimiento resultaron superiores a los lugares de origen, fueron elegidas como promisorias. Para evaluar su potencial de crecimiento se compararon las alturas dominantes observadas con las de tablas de producción a la misma edad e índice de sitio de esas especies en su área de distribución natural. Las especies que presentaron mayor crecimiento que en su lugar de origen, tienen maderas de calidad, reconocido valor en el mercado y resultaron promisorias fueron: Quercus robur, Pinus monticola, Pinus sylvestris y Larix decidua. Éstas demostraron un gran potencial de cultivo y se proponen para ensayos de procedencia y plantación para diversificar forestaciones.

Palabras clave: introducción de especies, diversificación, especies de valor, Patagonia.

\section{INTRODUCCIÓN}

En la precordillera patagónica argentina, entre el bosque y la estepa en las provincias de Neuquén, Río Negro y Chubut, existen más de dos millones de hectáreas aptas para el establecimiento de forestaciones (Gallo et al. 2005). Esa superficie corresponde a tierras ganaderas que, como consecuencia del sobrepastoreo por más de un siglo, presentan hoy diversos niveles de degradación (Soriano 1983, Broquen et al. 2003). En esta región, entre 
1945 y 1948, las Administraciones de Parques Nacionales y Bosques de Argentina llevaron a cabo pequeñas forestaciones con fines demostrativos, a las que se sumaron las realizadas por pioneros en algunos establecimientos rurales (Godoy 2000). Todas estas forestaciones se hicieron de manera empírica y sin diseños experimentales acordes, y debido a la falta de registros, tampoco se conoce la procedencia del material de reproducción (Enricci 1994). Sin embargo y a pesar de esa falta de información, estos pequeños rodales y grupos de árboles constituyen hoy un valioso muestrario de los resultados de dichas introducciones, que evaluados y analizados cuidadosamente, permitirían preseleccionar, sobre bases científicas, especies promisorias para futuras forestaciones. El contar con estos datos es de fundamental importancia para diversificar las plantaciones existentes, ya que en la actualidad sólo tres especies de coníferas son utilizadas en forestaciones masivas. De las 70 mil ha plantadas, el $80 \%$ corresponde a pino ponderosa (Pinus ponderosa (Dougl. ex Laws.)), mientras que el resto se reparte en proporciones similares entre pino oregón (Pseudotsuga menziesii (Mirb.) Franco) y pino contorta (Pinus contorta (Dougl.) var. Murrayana (Gallo et al. 2005). No existen aún datos sobre crecimiento, estado sanitario u otras características dasométricas y ambientales relacionadas que permitan inferir cuáles de las especies se presentan como promisorias para acompañar a las tres coníferas mencionadas en futuras plantaciones.

El objetivo de este trabajo fue: 1) identificar las especies exóticas en plantaciones (rodales y grupos de árboles) localizadas en el área de estudio, 2) describir dasométricamente los rodales y caracterizar su calidad, expresada en la forma de los fustes y su sanidad, y 3) seleccionar, en base a estos parámetros y a la comparación de su crecimiento en altura con el óptimo registrado en su lugar de origen, las especies consideradas como promisorias, describiendo además las principales características edafoclimáticas de los sitios en que se desarrollan.

\section{MÉTODOS}

Los sitios muestreados se encuentran en las provincias de Neuquén, Río Negro y Chubut, entre los parale-

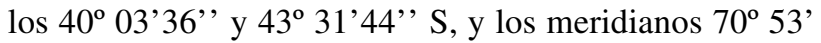
$41^{\prime}$ ' y $71^{\circ} 33^{\prime}, 58^{\prime}$ ' O. Las altitudes comprenden desde los 300 hasta los $1.270 \mathrm{~m}$ s.n.m. El clima es templado frío con régimen invernal de precipitaciones, siendo el verano una estación seca marcada, con vientos predominantes del cuadrante oeste (Dimitri 1982). La variación climática más importante son las precipitaciones, que disminuyen drásticamente de oeste a este, en un gradiente aproximado de $50 \mathrm{~mm} / \mathrm{km}$ (Barros et al. 1979). De esta manera, sitios como Estancia Quechuquina reciben $2.200 \mathrm{~mm} / \mathrm{año}$, mientras que en Estancia Chimehuin sólo $550 \mathrm{~mm} / \mathrm{año.}$
Al aumentar la latitud disminuyen las temperaturas y la duración del período seco, y por lo tanto la evapotranspiración potencial (Barros et al. 1979, Jobbágy et al. 1995). La topografía de todos los sitios de estudio es plana. El material originario del suelo es ceniza volcánica. Estos suelos taxonómicamente corresponden al orden de los Andisoles y Molisoles (Soil Survey Staff 1999). Los primeros se caracterizan por ser profundos, con una capacidad de retención hídrica mayor al 35\%, baja densidad aparente $\left(0,85 \mathrm{~g} / \mathrm{cm}^{3}\right)$ y reacción ligeramente ácida (Buduba 2006), con valores de materia orgánica en los primeros horizontes que abarcan un rango entre 6 y $11 \%$ (Colmet Daage 1993). Los segundos tienen reacción neutra o ligeramente ácida, con baja disponibilidad de agua en los horizontes superficiales pero mayor en profundidad, densidad aparente de 0,9 a $1,0 \mathrm{~g} / \mathrm{cm}^{3}$ y contenido de materia orgánica entre 3\% y 5\% (Buduba 2006). Los tipos de ceniza volcánica contenida son de mayor a menor capacidad de retención de agua: alófano, imogolita y haloisita (Irisarri 2000). La textura de los suelos donde se hallaron las forestaciones fueron franca arenosa a excepción del rodal 2 de alerce europeo en Cuesta del Ternero, que fue franca arcillosa. Una descripción a nivel de gran grupo (según el Soil Survey Staff 1999), así como de las características climáticas y la ubicación de cada sitio se presentan en el cuadro 1.

En cada rodal, grupo de árboles (< 100 árboles de una misma especie agrupados en un sitio) y/o árboles individuales se identificó la especie y se obtuvieron los parámetros dasométricos y de calidad. En cada rodal se estableció una parcela en la que se midió un mínimo de 100 árboles, excluyendo dos filas de árboles de borde. En el caso de los grupos de árboles se midieron todos los individuos. Las variables consideradas fueron: superficie (en hectáreas) en el caso de los rodales, y tanto en los rodales como en los grupos de árboles el diámetro a la altura del pecho DAP $(\mathrm{cm})$, altura total $(\mathrm{m})$ y la edad con barreno de Pressler. Los árboles se clasificaron en 1) sanos bien formados (rectos y sin evidencias de enfermedades), 2) sanos mal formados (tortuosos y/o bifurcados, sin evidencias de enfermedades), 3) enfermos y 4) muertos. La densidad se determinó sólo para los rodales, y no para los grupos de árboles porque el cálculo de la misma podría llevar a conclusiones erróneas.

Un buen indicador del potencial productivo de una especie en un sitio determinado es el desarrollo en altura de los árboles dominantes (Daniel et al. 1982, Hanson et al. 2001). Para evaluar la productividad de las especies consideradas como promisorias, se compararon las alturas dominantes de los rodales y las alturas máximas de los grupos de árboles -que a partir de ahora se unificará con el concepto de altura dominante-, con las que alcanza la misma especie en su lugar de origen a la misma edad. Para las especies de origen europeo, se utilizaron las tablas de producción de Schober (1987) desarrolladas en Alemania. Para Quercus robur se usaron además las 
Cuadro 1. Datos climáticos, edáficos y ubicación de los sitios muestreados. Climatic, edaphic and location data.

\begin{tabular}{|c|c|c|c|c|c|c|c|c|}
\hline $\begin{array}{c}\text { Datos climáticos, suelos } \\
\text { y ubicación }\end{array}$ & $\begin{array}{c}\text { Ea. } \\
\text { Quechuquina }\end{array}$ & $\begin{array}{c}\text { Co. } \\
\text { Chapelco }\end{array}$ & $\begin{array}{c}\text { Isla } \\
\text { Victoria }\end{array}$ & $\begin{array}{c}\text { INTA } \\
\text { Golondrinas }\end{array}$ & $\begin{array}{l}\text { INTA } \\
\text { Trevelin }\end{array}$ & $\begin{array}{l}\text { Cuesta del } \\
\text { Ternero }\end{array}$ & $\begin{array}{l}\text { Co. del } \\
\text { los Pinos }\end{array}$ & $\begin{array}{c}\text { Ea. } \\
\text { Chimehuin }\end{array}$ \\
\hline Precipitaciones medias anuales $(\mathrm{mm})$ & 2200 & 1358 & 1300 & 972 & 952 & 750 & 650 & 550 \\
\hline Temperatura media anual $\left({ }^{\circ} \mathrm{C}\right)$ & 9,4 & 10,9 & 8 & 10,0 & 9,8 & $\mathrm{~s} / \mathrm{d}^{*}$ & $10-12$ & $10-12$ \\
\hline $\begin{array}{l}\text { Clasificación Suelo Gran Grupo } \\
\text { Tipo de ceniza }\end{array}$ & $\begin{array}{l}\text { Udivitrand } \\
\text { alófano }\end{array}$ & $\begin{array}{l}\text { Udivitrand } \\
\text { alófano }\end{array}$ & $\begin{array}{l}\text { Udivitrand } \\
\text { alófano }\end{array}$ & $\begin{array}{l}\text { Udivitrand } \\
\text { alófano }\end{array}$ & $\begin{array}{l}\text { Udivitrand } \\
\text { imogolita }\end{array}$ & $\begin{array}{l}\text { Argixerol } \\
\text { haloisita }\end{array}$ & $\begin{array}{l}\text { Argixerol } \\
\text { haloisita }\end{array}$ & $\begin{array}{c}\text { Haploxerol } \\
\text { haloisita }\end{array}$ \\
\hline Profundidad del suelo $(\mathrm{cm})$ & 113 & 71 & 115 & 98 & 90 & 70 & 100 & 124 \\
\hline Altitud (m snm) & 1270 & 1000 & 759 & 320 & 270 & 800 & 740 & $835 \mathrm{~m}$ \\
\hline Latitud sur & $40^{\circ} 05^{\prime} 24^{\prime \prime}$ & $40^{\circ} 09^{\prime} 05^{\prime \prime}$ & $40^{\circ} 51^{\prime} 11^{\prime \prime}$ & $42^{\circ} 00^{\prime} 00^{\prime \prime}$ & $43^{\circ} 011^{\prime} 48^{\prime \prime}$ & $41^{\circ} 56^{\prime} 12^{\prime \prime}$ & $40^{\circ} 03^{\prime} 36^{\prime \prime}$ & $40^{\circ} 06^{\prime} 28^{\prime \prime}$ \\
\hline Longitud oeste & $71^{\circ} 06^{\prime} 36^{\prime \prime}$ & $71^{\circ} 21^{\prime} 04^{\prime \prime}$ & $71^{\circ} 30^{\prime} 05^{\prime \prime}$ & $71^{\circ} 16^{\prime} 48^{\prime \prime}$ & $71^{\circ} 16^{\prime} 48^{\prime \prime}$ & $71^{\circ} 23^{\prime} 05^{\prime \prime}$ & $71^{\circ} 06^{\prime} 36^{\prime \prime}$ & $70^{\circ} 53^{\prime} 41^{\prime \prime}$ \\
\hline
\end{tabular}

$*_{\mathrm{s}} / \mathrm{d}=$ sin datos, no data.

curvas de índice de sitio de la especie según Barrio Anta et al. (2002), confeccionadas para Lugo en España. Para Pinus monticola se emplearon las curvas de índice de sitio de Curtis et al. (1990) correspondientes a bosques naturales de esa especie en la cadena montañosa Cascade Range de Washington y Oregon, Estados Unidos.

Las alturas dominantes utilizadas fueron:

- $\mathrm{h}_{100}$ : altura dominante según Assmann: altura media de los 100 árboles más gruesos por hectárea (Assmann 1970), que se determinó para comparar la productividad de todas las especies evaluadas,

- $\mathrm{h}_{\mathrm{w}}$ : altura dominante según Weise: altura media del $20 \%$ de los árboles con mayor área basal (Smith et al. 1997), que se utilizó sólo para roble europeo porque es la que presenta la tabla de Schober (1987) para dicha especie.

Con los resultados dasométricos de las plantaciones y la comparación de las productividades entre Patagonia y los lugares de origen, se seleccionaron las especies promisorias. Los criterios de selección fueron: que la especie estuviera representada por lo menos en dos sitios en forma de rodal, que el $95 \%$ o más de los individuos vivos de esos rodales o grupos de árboles estuvieran sanos, que el número de individuos muertos por competencia no superara el $12 \%$ y que la altura dominante alcanzada fuera igual o mayor que la alcanzada por la misma especie en su lugar de origen a una misma edad, para las mejores calidades de sitio.

\section{RESULTADOS}

Se identificaron en total 54 especies (cuadro 2) pertenecientes a nueve familias de latifoliadas y tres de coníferas. La mayoría de las latifoliadas están representadas con un género por familia y una o dos especies por géne-

Cuadro 2. Especies identificadas en rodales, grupos de árboles y árboles individuales.

Identified species in stands, groups of trees and individual trees.

Género Especies

Abies

Araucaria

Betula

Cedrus

Cupressus

Eucalyptus

Fraxinus

Juglans

Larix

Nothofagus

Picea

Pinus

Populus

Prunus

Quercus

Robinia

Sequoia

Sequoiadendron

Tilia

Thuja

Ulmus
A. alba Mill., A. grandis Lindley, A. lasiocarpa (Hook) Nutt., A. magnifica A. Murr., A. pinsapo Boiss.

A. araucana (Mol.) Koch

B. albo-sinensis Buró, B. pendula Roth.

$C$. deodara Laws., C. libani Laws.

C. arizonica Greene, C. macrocarpa Gord., C. torulosa D. Don

E dalrympleana Maiden, E. fastigata Deane y Maiden, E. johnstonii Maiden, E. ovata Labill., E. regnans F. Muell., E. viminalis Labill.

F. excelsior $L$.

J. regia $\mathrm{L}$.

L. decidua Mill., L. kaempferi (Lamb) Carr. N. obliqua (Mirb.) Blume P. abies (L.) Karst., P. sitchensis (Bong.) P. banksiana Lamb., P. cembra L., P. coulteri Dougl. ex D. Don., $P$. jeffreyi Balf, $P$. lambertiana Dougl., $P$. monticola Dougl. ex D. Don, P. nigra Arnold, P. pinaster Ait., $P$. radiata D. Don., $P$. resinosa Ait., $P$. rigida Mill., P. sabiniana Dougl., P. strobus L., $P$. sylvestris L., P. thunbergii Parlatore, $P$. $x$ euroamericana (Dode) Guinier, $P$. nigra L., P. trichocarpa Torr. y Gray

$P$. avium $\mathrm{L}$.

Q. macrocarpa Michx., Q. robur $\mathrm{L}$.

$R$. pseudoacacia $\mathrm{L}$.

S. sempervirens (D. Don) Endl.

S. giganteum (Lindl.) Buchholz

T. plathyphyllos Scop.

T. giganteum Donn ex D. Donn, T. occidentalis $\mathrm{L}$.

U. pumila L. 
ro (con excepción del género Eucalyptus). Las coníferas, en cambio, están representadas por más de un género y especie por familia (con excepción de Araucaria araucana), con 15 especies del género Pinus. De todas las especies identificadas, las promisorias fueron: $Q$. robur, $P$. monticola, Pinus sylvestris y Larix decidua.

A continuación se detallan los parámetros dasométricos y ambientales relevantes.

Quercus robur. En todos los casos, las alturas dominantes (según Weise) superaron a las reportadas por Schober (1987) para la mejor calidad de sitio en Alemania (cuadro 3 y figura 1). También las alturas dominantes locales (según Assmann) fueron superiores a las curvas provistas para España por Barrio Anta et al. (2002) para el índice de sitio más alto, salvo en los casos de Estancia Quechuquina e INTA Trevelin (cuadro 3).

El rango de precipitaciones en el que crecieron estos rodales varía entre $550 \mathrm{~mm} /$ año en Estancia Chimehuin y $2.200 \mathrm{~mm} / \mathrm{año}$ en Estancia Quechuquina. Llama la atención la altura dominante en Estancia Chimehuin, que alcanzó 24,6 m a los 50 años. En ese lugar se hallaron antiguos canales de riego, actualmente abandonados, que muy probablemente suplieron de agua a ese rodal durante sus etapas tempranas de crecimiento. No se observó una tendencia clara entre la precipitación y la altura dominante alcanzada (figura 2). También se destaca la amplitud de características de suelos, como Haploxerols en Estancia Chimehuin, y Argixerols en Cerro de los
Pinos (con contenido de haloisita) y Udivitrands en Estancia Quechuquina, INTA Trevelin e INTA Golondrinas (con contenido de imogolita y alófano) (cuadro 1). Aunque ningún rodal recibió manejo silvicultural, se destacó en general la rectitud de fustes. Así, en Estancia Chimehuin se encontraron el $100 \%$ de los árboles bien formados, en INTA Golondrinas el 86\%, en INTA Trevelin el 63\%, en Cerro de los Pinos el $40 \%$ en ambos rodales y en Estancia Quechuquina sólo el 20\% tuvo buena forma. Las edades de estas plantaciones variaron entre los 26 y 50 años de edad, y los diámetros medios cuadráticos (DMC) fueron entre $\operatorname{los} 19$ y $38 \mathrm{~cm}$, no encontrándose relación entre la densidad y el diámetro a edades similares (cuadro 3).

Pinus monticola. La altura dominante de esta especie fue mayor en todos los lugares muestreados en Patagonia (cuadro 4, figura 1) que la del mejor sitio en bosques nativos en Estados Unidos (curva de índice de sitio 30,4 m a los 50 años) que reporta Curtis et al. (1990). La excepción fue la de Isla Victoria, que resultó levemente menor a la de la curva mencionada. A esta especie se la encontró creciendo entre los $970 \mathrm{~mm} / \mathrm{año}$ de precipitación en INTA Golondrinas hasta los $2.200 \mathrm{~mm} / \mathrm{año}$ en Estancia Quechuquina, con suelos clasificados como Udivitrands, que tienen alto contenido de cenizas volcánicas del tipo alófano, y muy profundos (cuadro 1). En sitios con precipitación superior a los $1.200 \mathrm{~mm}$, se observa con el aumento de las precipitaciones un incremento de la altura dominante.

Cuadro 3. Características de seis rodales o grupos de árboles de Quercus robur en Patagonia, y alturas dominantes para los mejores sitios en Alemania y España.

Characteristics of six Quercus robur stands or groups of trees and dominant heights in Germany and Spain.

\begin{tabular}{|c|c|c|c|c|c|c|c|c|}
\hline \multirow[b]{2}{*}{ Lugar } & \multicolumn{6}{|c|}{ Patagonia $^{\mathrm{a}}$} & \multirow{2}{*}{$\begin{array}{c}\mathrm{h}_{\mathrm{w}}{ }^{\mathrm{b}} \\
(\mathrm{m}) \\
\text { Alemania }\end{array}$} & \multirow{2}{*}{$\begin{array}{c}\mathrm{h}_{100}{ }^{\mathrm{c}} \\
(\mathrm{m}) \\
\text { España }\end{array}$} \\
\hline & $\begin{array}{l}\text { Sup. } \\
\text { o N }^{\circ}\end{array}$ & $\begin{array}{l}\text { Edad } \\
\text { (años) }\end{array}$ & N/ha & $\begin{array}{c}\text { DMC } \\
(\mathrm{cm})\end{array}$ & $\begin{array}{l}\mathrm{h}_{100} \\
(\mathrm{~m})\end{array}$ & $\begin{array}{l}\mathrm{h}_{\mathrm{w}} \\
(\mathrm{m})\end{array}$ & & \\
\hline Ea. Quechuquina & 0,2 ha & 50 & 500 & 37,8 & 22,4 & 22,4 & 19,7 & 23,0 \\
\hline Ea. Chimehuin & 2,0 ha & 50 & 866 & 25,8 & 24,6 & 24,6 & 19,7 & 23,0 \\
\hline INTA Golondrinas & 0,25 ha & 46 & 1.285 & 24,2 & 22,0 & 22,3 & 18,7 & 21,2 \\
\hline INTA Trevelin & 0,3 ha & 45 & 1.100 & 27,5 & 20,5 & 20,7 & 18,5 & 21,0 \\
\hline Co. de los Pinos (rodal 2) & 32 árb. & 30 & - & $23,5^{\mathrm{d}}$ & 18,5 & 18,8 & 14,1 & 14,0 \\
\hline Co. de los Pinos (rodal 1) & 27 árb. & 26 & - & $17,3^{\mathrm{d}}$ & 13,8 & 14,0 & 12,3 & 12,5 \\
\hline
\end{tabular}

a Sup.: superficie del rodal. $\mathrm{N}^{\mathrm{o}}$ : número de árboles del grupo. $\mathrm{N} /$ ha: número de árboles por hectárea. DMC $=$ diámetro medio cuadrático. $\mathrm{h}_{100}=$ altura dominante de Assmann, $\mathrm{h}_{\mathrm{w}}=$ altura dominante de Weise.

$\mathrm{h}_{\mathrm{w}}=$ según Schober (1987).

$\mathrm{h}_{100}=$ según Barrio Anta et al. (2002), ambas para la misma edad que el rodal o grupo de árboles en Patagonia.

corresponde a diámetro altura de pecho DAP promedio.

Sup.: surface area. $\mathrm{N}^{\mathrm{o}}$ : number of trees of the group. $\mathrm{N} / \mathrm{ha}$ : number of trees per hectare. DMC $=$ mean quadratic diameter; $\mathrm{h}_{100}=\mathrm{dominant}$ height of Assmann; $h_{w}=$ dominant height of Weise.

$h_{w}$ according to Schober (1987).

c $\mathrm{h}_{100}$ according to Barrio Anta et al. (2002), both heights for the same age that has the stand or group of trees in Patagonia.

d These data correspond to average diameter at height breast DHB. 

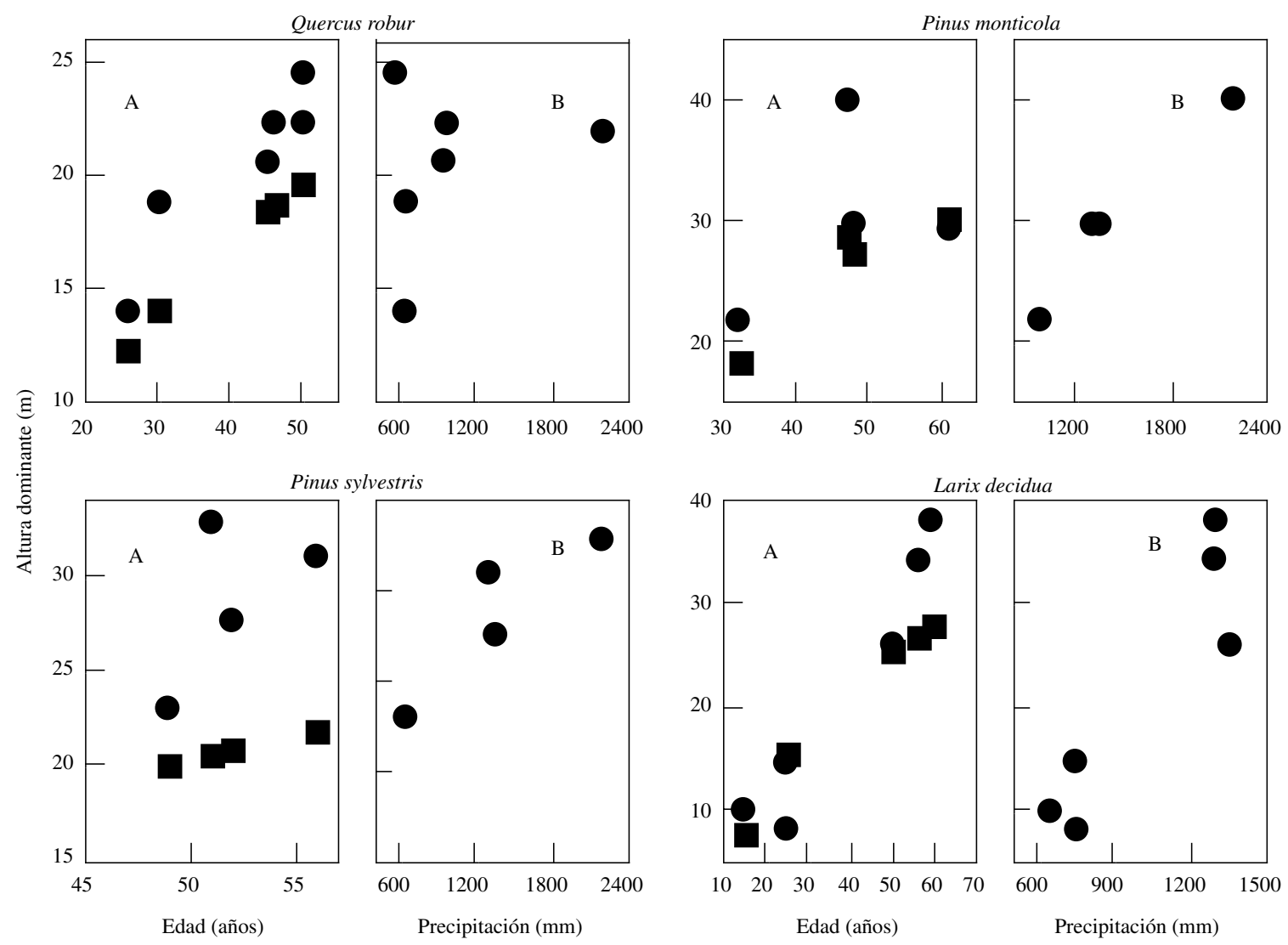

Figura 1. Alturas dominantes de diferentes especies: A) medidas en Patagonia (círculos) y en el lugar de origen según Schober (1987) (cuadrados) para Quercus robur, Pinus sylvestris y Larix decidua, y según Curtis et al. (1990) para Pinus monticola; B) en relación a las precipitaciones anuales.

Dominant heights of different species: A) measured in Patagonia (circles) and in their original places according to Schober (1987) (squares) for Quercus robur, Pinus sylvestris and Larix decidua, and according to Curtis et al. (1990) for Pinus monticola; $B$ ) related to annual precipitations.

Cuadro 4. Características de cuatro rodales o grupos de árboles de Pinus monticola en Patagonia y en los mejores sitios en las Cascade Range, EE.UU.

Characteristics of four Pinus monticola stands or groups of trees and dominant heights in the Cascade Range, USA.

\begin{tabular}{|c|c|c|c|c|c|c|}
\hline \multirow[b]{2}{*}{ Lugar } & \multicolumn{5}{|c|}{ Patagonia $^{\mathrm{a}}$} & \multirow{2}{*}{$\begin{array}{c}\mathrm{h}_{100}{ }^{\mathrm{b}} \\
(\mathrm{m}) \\
\text { E.E.U.U }\end{array}$} \\
\hline & $\begin{array}{l}\text { Sup. } \\
\text { o No }\end{array}$ & $\begin{array}{l}\text { Edad } \\
\text { (años) }\end{array}$ & N/ha & $\begin{array}{l}\text { DMC } \\
(\mathrm{cm})\end{array}$ & $\begin{array}{l}\mathrm{h}_{100} \\
(\mathrm{~m})\end{array}$ & \\
\hline Isla Victoria & 0,2 ha & 61 & 6.000 & 20,5 & 29,5 & 30,4 \\
\hline Co. Chapelco & 12 árb. & 48 & - & $44,4^{\mathrm{c}}$ & 29,6 & 28,4 \\
\hline Ea. Quechuquina & 8 árb. & 47 & - & $65,0^{\mathrm{c}}$ & 40,0 & 27,9 \\
\hline INTA Golondrinas & 0,6 ha & 32 & 1.000 & 21,1 & 21,8 & 18,3 \\
\hline
\end{tabular}

a Sup.: superficie del rodal. $\mathrm{N}^{\mathrm{o}}$ : número de árboles del grupo. $\mathrm{N} / \mathrm{ha}$ : número de árboles por hectárea. $\mathrm{DMC}=$ diámetro medio cuadrático. $\mathrm{h}_{100}=$ altura dominante de Assmann.

b $\mathrm{h}_{100}$ en Curtis et al. (1990) a la misma edad que el rodal o grupo de árboles en Patagonia.

c corresponde a diámetro altura de pecho DAP promedio.

a Sup.: surface area. $\mathrm{N}^{\mathrm{o}}$ : number of trees of the group. N/ha: number of trees per hectare. DMC $=$ mean quadratic diameter; $\mathrm{h}_{100}=$ dominant height of Assmann.

b $\mathrm{h}_{100}$ according to Curtis et al. (1990) at the same age than the stand or group of trees in Patagonia.

c These data correspond to average diameter at height breast DHB.
Todos los árboles de esta especie presentaron en general muy buena forma del fuste. La frecuencia de individuos bien formados fue del $100 \%$ en INTA Golondrinas, $92 \%$ en Cerro Chapelco, $87 \%$ en Estancia Quechuquina y 66\% en Isla Victoria. La edad de los rodales varió entre 32 y 61 años de edad y los DMC alcanzaron entre 21 y $65 \mathrm{~cm}$.

Pinus sylvestris. Este pino también superó en todos los rodales y grupos de árboles en Patagonia la altura dominante de la mejor calidad de sitio en Alemania, según Schober (1987) (cuadro 5, figura 1). La calidad de sitio mejora con la precipitación (figura 1) de tal modo que, alrededor de los 50 años de edad, donde la precipitación anual es de $650 \mathrm{~mm}$, la altura dominante es de $23 \mathrm{~m}$, mientras que donde precipitan $2.200 \mathrm{~mm}$ anuales la altura dominante es de $33 \mathrm{~m}$.

Respecto de las condiciones ambientales, esta especie se encontró creciendo en un amplio rango de precipitaciones, desde los 650 a los $2.200 \mathrm{~mm} / \mathrm{año}$, en suelos profundos, en Argixeroles con haloisita en Cerro de los 
Cuadro 5. Características de cuatro rodales o grupos de árboles de Pinus sylvestris en Patagonia y alturas dominantes en los mejores sitios en Alemania.

Characteristics of Pinus sylvestris stands and groups of trees and dominant heights in Germany.

\begin{tabular}{|c|c|c|c|c|c|c|}
\hline \multirow[b]{2}{*}{ Lugar } & \multicolumn{5}{|c|}{ Patagonia $^{\mathrm{a}}$} & \multirow{2}{*}{$\begin{array}{c}\mathrm{h}_{100}{ }^{\mathrm{b}} \\
(\mathrm{m}) \\
\text { Alemania }\end{array}$} \\
\hline & $\begin{array}{l}\text { Sup. } \\
\text { o N }\end{array}$ & $\begin{array}{l}\text { Edad } \\
\text { (años) }\end{array}$ & $\mathrm{N} / \mathrm{ha}$ & $\begin{array}{l}\text { DMC } \\
(\mathrm{cm})\end{array}$ & $\begin{array}{l}\mathrm{h}_{100} \\
\text { (m) }\end{array}$ & \\
\hline Isla Victoria & 37 árb. & 56 & - & $36,1^{\mathrm{c}}$ & 31,0 & 21,9 \\
\hline Cerro Chapelco & 20 árb. & 52 & - & $41,0^{\mathrm{c}}$ & 27,7 & 20,9 \\
\hline Ea. Quechuquina & $1,5 \mathrm{ha}$ & 51 & 265 & 44,8 & 32,9 & 20,6 \\
\hline Co. de los Pinos & 0,1 ha & 49 & 900 & 36,3 & 23,0 & 20,1 \\
\hline
\end{tabular}

a Sup.: superficie del rodal. $\mathrm{N}^{\mathrm{o}}$ : número de árboles. N/ha: número de árboles por hectárea. DMC = diámetro medio cuadrático. $\mathrm{h}_{100}=$ altura dominante de Assmann.

b $\quad h_{100}$ según Schober (1987) a la misma edad que el rodal o grupo de árboles en Patagonia.

corresponde a diámetro altura de pecho DAP promedio.

a Sup.: surface area. $\mathrm{N}^{\mathrm{o}}$ : number of trees of the group. $\mathrm{N} / \mathrm{ha}$ : number of trees per hectare. DMC $=$ mean quadratic diameter; $\mathrm{h}_{100}=$ dominant height of Assmann.

b $\mathrm{h}_{100}$ according to Schober et al. (1987) at the same age than the stand or group of trees in Patagonia.

c These data correspond to average diameter at height breast DHB.

Pinos y Udivitrands con alófano en Isla Victoria, Cerro Chapelco y Estancia Quechuquina (cuadros 1 y 4). La calidad de los fustes fue variable, con $100 \%$ de árboles bien formados en Cerro de los Pinos, 55\% en Cerro Chapelco, 38\% en Quechuquina y 30\% en Isla Victoria. En este último sitio se halló un $21 \%$ de individuos sin la parte superior de la copa, probablemente por efecto del viento, que allí es muy intenso, ya que esta especie es susceptible a daños provocados por este agente atmosférico (Skilling 1990). Con edades entre 49 y 51 años, el pino silvestre alcanzó DMC entre 36 y $45 \mathrm{~cm}$ (cuadro 5).

Larix decidua. Las alturas dominantes del alerce europeo en Patagonia superaron a las registradas en Alemania para la mejor calidad de sitio que cita Schober (1987), con excepción de los rodales en Cuesta del Ternero (cuadro 6, figura 1). En dicho sitio el rodal 1 correspondió a una calidad de sitio II, y la del rodal 2 a una calidad de sitio III, también según tablas de producción de Schober (1987).

Se la encontró en sitios con precipitación entre los 650 (en Cerro de los Pinos) y $1.358 \mathrm{~mm} / \mathrm{año}$ (en Cerro Chapelco) y en distintos suelos como Argixerols y Udivitrands, que contienen haloisita y alófano, respectivamente. Con precipitaciones mayores de $1.200 \mathrm{~mm} / \mathrm{año}$, se observaron las mayores alturas dominantes, con valores entre 26 y $38 \mathrm{~m}$ y edades entre 50 y 60 años, respectivamente (figura 1). La forma de los fustes fue variable, siendo la frecuencia de individuos bien formados en Isla Victoria del 76\%, en Cuesta del Ternero en ambos rodales el 53\%, en Cerro de los Pinos el 46\%, y como excep-
Cuadro 6. Características de seis rodales o grupos de árboles de Larix decidua en Patagonia y alturas dominantes para los mejores sitios en Alemania.

Characteristics of Larix decidua stands and groups of trees and dominant heights in Germany.

\begin{tabular}{|c|c|c|c|c|c|c|}
\hline \multirow[b]{2}{*}{ Lugar } & \multicolumn{5}{|c|}{ Patagonia $^{\mathrm{a}}$} & \multirow{2}{*}{$\begin{array}{c}\mathrm{h}_{100} \mathrm{~b} \\
(\mathrm{~m}) \\
\text { Alemania }\end{array}$} \\
\hline & $\begin{array}{l}\text { Sup. } \\
\text { o } \mathrm{N}^{\circ}\end{array}$ & $\begin{array}{l}\text { Edad } \\
\text { (años) }\end{array}$ & N/ha & $\begin{array}{l}\text { DMC } \\
(\mathrm{cm})\end{array}$ & $\begin{array}{l}\mathrm{h}_{100} \\
(\mathrm{~m})\end{array}$ & \\
\hline Isla Victoria & 31 árb. & 59 & - & $37,3^{\mathrm{d}}$ & 38,0 & 27,8 \\
\hline Isla Victoria $^{c}$ & 42 árb. & 56 & - & $38,1^{\mathrm{d}}$ & 34,2 & 28,0 \\
\hline Co. Chapelco & 78 árb. & 50 & - & $35,7^{\mathrm{d}}$ & 26,1 & 25,3 \\
\hline $\begin{array}{l}\text { Cuesta del Ternero } \\
\text { (rodal 1) }\end{array}$ & 0,1 ha & 25 & 1.000 & 19,7 & 14,6 & 15,4 \\
\hline $\begin{array}{l}\text { Cuesta del Ternero } \\
\text { (rodal 2) }\end{array}$ & 0,1 ha & $\begin{array}{l}25 \\
15\end{array}$ & $\begin{array}{c}2.227 \\
-\end{array}$ & $\begin{array}{l}14,4 \\
25,0^{\mathrm{d}}\end{array}$ & $\begin{array}{l}8,0 \\
9,9\end{array}$ & $\begin{array}{r}15,4 \\
7,8\end{array}$ \\
\hline
\end{tabular}

a Sup.: superficie del rodal. $\mathrm{N}^{\circ}$ : número de árboles del grupo. $\mathrm{N} /$ ha: número de árboles por hectárea. $\mathrm{DMC}=$ diámetro medio cuadrático, $\mathrm{h}_{100}=$ altura dominante de Assmann.

b $\mathrm{h}_{100}$ según Schober (1987) a la misma edad que el rodal o grupo de árboles en Patagonia.

c Rodal mixto que está conformado por Pseudotsuga menziesii, Pinus sylvestris, Pinus strobus y Picea sitchensis.

d Corresponde a diámetro altura de pecho DAP promedio.

a Sup.: surface area. $\mathrm{N}^{\circ}$ : number of trees of the group. $\mathrm{N} / \mathrm{ha}$ : number of trees per hectare. $\mathrm{DMC}=$ mean quadratic diameter; $\mathrm{h}_{100}=$ dominant height of Assmann.

b $\mathrm{h}_{100}$ according to Schober (1987) at the same age than the stand or group of trees in Patagonia.

c The mixed stand in Isla Victoria is conformed with Pseudotsuga menziesii, Pinus sylvestris, Pinus strobus and Picea sitchensis.

d These data correspond to average diameter at height breast DHB.

ción en Cerro Chapelco sólo el 10\% de los árboles, ya que la mayoría se encontraba combada a los $10 \mathrm{~m}$ de altura. También aquí el $11 \%$ de los individuos se halló muerto, probablemente debido a la alta competencia intraespecífica, aunque el estado sanitario fue muy bueno. Esta especie se halló en rodales y grupos de árboles cuyas edades variaron entre 15 y 59 años y sus DMC entre 14 y $37 \mathrm{~cm}$.

\section{DISCUSIÓN}

La productividad, considerando como tal las alturas dominantes alcanzadas, de las especies estudiadas y definidas como promisorias en Patagonia, fue en su mayoría superior a la registrada tanto en bosques naturales como en plantaciones en sus lugares de origen. Es probable que los suelos en la Patagonia andina, por su profundidad y alto contenido en cenizas volcánicas, con capacidad de retener agua disponible para las plantas y proveerla en las etapas de crecimiento durante el período seco de primavera-verano, jueguen un rol importante en la productividad de estas cuatro especies.

Como otro ejemplo de la notable diferencia de mayor productividad, según Mayer (1992), Q. robur, a los 150 
años de edad, alcanza en los mejores sitios en Europa central 25-30 m de altura. En Patagonia, a los 45-50 años, alcanzó alturas dominantes de 20 a $24 \mathrm{~m}$, lo cual significa que el turno de esta especie se adelantaría considerablemente. El desarrollo sobresaliente comprobado en la Estancia Chimehuin (550 mm/año) puede haber estado influenciado al comienzo de su desarrollo por riego artificial, lo cual indica que la especie podría adaptarse a condiciones xéricas (menores a 900 mm/año), lo que debería probarse.

Para P. monticola, Guerra et al. (1988) citan que en Laguna Breham, Provincia del Chubut, Argentina, con una precipitación media anual de $700 \mathrm{~mm}$, el árbol promedio midió 22,9 $\mathrm{m}$ de altura a los 28 años de edad, valor similar a la altura dominante hallada en INTA Golondrinas a los 32 años de edad del rodal y $970 \mathrm{~mm} /$ año. Haig (1932) y Graham (1990) señalan que los mejores sitios para $P$. monticola presentan entre 18 y $24 \mathrm{~m}$ de altura a los 50 años de edad respectivamente, valores que son superados en Patagonia incluso a menor edad en los rodales encontrados en Cerro Chapelco y Estancia Quechuquina.

Pinus sylvestris muestra un índice de sitio de $24 \mathrm{~m} \mathrm{de}$ altura a los 50 años en Suiza, lo que se considera muy bueno (Duc 2001). Sin embargo, este valor es superado en Patagonia alrededor de esa edad, como en los rodales que se encuentran en sitios con precipitaciones mayores a los $1.300 \mathrm{~mm} / \mathrm{año} \mathrm{(Estancia} \mathrm{Quechuquina,} \mathrm{Isla} \mathrm{Victoria}$ y Cerro Chapelco), y que presentan suelos con contenido de alófano, ceniza con alta capacidad de retención de agua. Ese mismo autor indica $22,5 \mathrm{~m}$ de altura a los 50 años como índice de sitio bueno, valor comparable al obtenido en Cerro de los Pinos, con precipitación media de $650 \mathrm{~mm} / \mathrm{año}$. La diferencia de altura dominante entre los rodales de Isla Victoria y Cerro Chapelco podría deberse a diversos factores, entre los que merece destacarse la profundidad de los suelos (aproximadamente el 30\% más en Isla Victoria).

Larix decidua llega a $27 \mathrm{~m}$ de altura a los 50 años en Suiza (Duc, 2001), como índice de sitio muy bueno, valor muy próximo a los alcanzados en los sitios más húmedos analizados, como en Isla Victoria y Cerro Chapelco. Glen e Isherwood (1999) citan como índice de sitio medio $19 \mathrm{~m}$ de altura a los 50 años en plantaciones en Canadá, valor que es sobrepasado ampliamente en los sitios en Patagonia antes mencionados. La excepción del rodal 2 en Cuesta del Ternero es debida a que éste se encontraba en una parte baja del terreno que antes de la plantación habría sido un mallín (Gomis 1973), actualmente seco. Como es sabido, los mallines son áreas con suelos pesados, con textura franca arcillosa, sin haloisita y que permanecen anegados una gran parte del año (Berasategui 1998, San Martino 2003). Ello pudo haber retrasado el crecimiento del L. decidua en este sitio. Esta especie se desarrolla mejor en suelos profundos (Mayer 1992). Como en el caso de $P$. sylvestris, la diferencia de alturas dominantes entre Cerro Chapelco e Isla Victoria podrían estar dadas por la profundidad del suelo, que es un $30 \%$ mayor en el último (cuadro 1 ).

\section{CONCLUSIONES}

En este trabajo se identificaron 54 especies que crecen en distintos lugares en la Patagonia argentina. De éstas sólo cuatro se presentaron como promisorias para ser seleccionadas en ensayos de procedencia y plantación en Patagonia por su adaptación a las condiciones generales de clima y suelo de los sitios en que se encuentran y por su buen nivel de crecimiento. El resto precisa aún demostrar su adaptación al suelo y clima de la región mediante ensayos de introducción en distintos sitios.

\section{REFERENCIAS}

Assmann E. 1970. The principles of forest yield study. Traducido por SH Gardiner y PW Davis. Oxford, Pergamon Press. 506 p.

Barrio Anta M, IJ Díaz-Maroto, JG Álvarez-González, MA Balboa Murias. 2002. Curvas de calidad para Quercus robur L. en el norte de la provincia de Lugo. Depto. de Enxeñería Agroforestal. Escola Politécnica Superior, Universidad de Santiago de Compostela. Consultado 30 nov. 2003. Disponible en: http://www.juntadeandalucia.es/ medioambiente/ ponencias/135.htm

Barros V, B Scian, H Matto. 1979. Campos de precipitación en la provincia de Chubut (período 1931-1960). Geoacta 10: 175-192.

Berasategui L. 1998. Clasificación de suelos. In Conti M ed. Principios de edafología con énfasis en suelos argentinos. Buenos Aires, Orientación Gráfica Editora. p. 5-20.

Broquen P, G Falbo, A Apcarián, F Candan, J Girardin, V Pellegrini. 2003. Relaciones entre las forestaciones, la erosión del suelo y la potencialidad productiva en la transición bosque-estepa (Andinopatagonia, Argentina). Invest. Agrar.: Sist. Recur. For. (España) 12 (2): 99-100.

Buduba CG. 2006. Modificaciones en el pH y contenido de materia orgánica en suelos del ecotono estepa/bosque andino-patagónico por implantación de pino ponderosa. Tesis de Doctorado. Facultad de Agronomía, Universidad de Buenos Aires. 201 p.

Colmet Daage F. 1993. Principales áreas forestales de la Argentina. Productividad elevada y sostenida: dos caracteres favorables en el Cono Sur. Suelos forestales 10: 257-259.

Curtis RO, NM Díaz, GW Clendenen. 1990. Heigth growth and site index curves for western white pine in the Cascade Range of Washington and Oregon. Res. Pap. PNW-RP-423. Portland, OR:US. Department of Forest Service, Pacific Northwest Research Station. 14 p.

Daniel TW, JA Helms, FS Baker. 1982. Principles of silviculture. $2^{\mathrm{a}}$ edición. New York, McGraw-Hill. 500 p.

Dimitri MJ. 1982. La región de los bosques andino-patagónicos. Tomo II. Colección Científica del INTA, Buenos Aires, Argentina, 179 p. 
Duc P. 2001. Sustainable forest regeneration. In Brassel P, H Liscke eds. Swiss National Forest Inventory: Methods and models of the second assessment. Wirmensdorf, Swiss Federal Research. p. 207-224.

Enricci JA. 1994. Posibilidades para la forestación en la subregión central del ecosistema andino patagónico. Buenos Aires, Argentina, CIEFAP. Publicación Técnica 14. Segunda Edición, 109 p.

Gallo L, A Martínez Maier, MM Azpillicueta, P Marchelli, V Mondino. 2005. Subprograma coníferas y otras especies en la región patagónica. In Norverto CA ed. Mejores árboles para más forestadores. Secretaría de Agricultura, Ganadería, Pesca y Alimentos, Buenos Aires, Argentina. p. 95-115.

Glen WM, HE Isherwood. 1999. Management Notes, № 22. Charlottetown, Estados Unidos, Department of Energy and Forestry, Forestry Division. 9 p.

Godoy MM. 2000. Especies forestales alternativas. Ficha Técnica. Revista Patagonia Forestal año 6 (2): 7-10.

Gomis H. 1973. Ensayos forestales en la región subandina de Río Negro. Dirección de Recursos Forestales Renovables. Ministerio de Economía, Prov. de Río Negro, Argentina. $26 \mathrm{p}$.

Graham RT. 1990. Western white pine. In Burns, R.M. y Honkala, B.H. Eds. Silvics of North America. Volume 1, Conifers. Agriculture Handbook 654. Forest Service, United States Department of Agriculture, Washington, DC, Estados Unidos. p. 385-394.

Guerra PE, G Cortés, NM Pasquini, J Lomagno. 1988. Posibilidades del cultivo de Pinus monticola (Dougl.) en el oeste del Chubut. Tomo II del VI Congreso Forestal Argentino 1988. Santiago del Estero, Argentina. p. 353-355.

Haig IT. 1932. Second-growth yield, stand and volume tables for the western white pine type. Tech. Bulletin $N^{\circ} 323$. U.S. Dept. of Agriculture. Washington D.C. U.S. Department of Agriculture. $67 \mathrm{p}$.
Hanson EJ, DL Azuma, BA Hiserote. 2001. Site Index Equations and Mean Annual Increment Equations for Pacific Northwest Research Station Forest Inventory and Analysis Inventories, 1985- Research Note PNW-RN-533. Pacific Northwest Research Station. USDA Forest Service. 25 p.

Irisarri JA. 2000. La propuesta de reclasificación de los Andes de Argentina, de acuerdo al Orden Andisoles. Workshop Soil Taxonomy. Buenos Aires, Argentina, INTA, AICET, AACS. p. 18-27.

Jobbágy EG, JM Paruelo, RJC León. 1995. Estimación del régimen de precipitación a partir de la distancia a la cordillera en el noroeste de la Patagonia. Ecología Austral 5: 47-53.

Mayer H. 1992. Walbau auf soziologisch-ökologischer Grundlage. $4^{\mathrm{a}}$ edición. Alemania, Gustav Fischer. 522 p.

San Martino L. 2003. Fertilización de mallines en la Patagonia. In Montes L ed. EEA-INTA Santa Cruz, Argentina. 44 p.

Schober R. 1987. Ertragstafeln wichtiger Baumarten. $3^{\text {a }}$ edición. Frankfurt am Main, Alemania. Sauerländer's Verlag. $166 \mathrm{p}$.

Skilling DD. 1990. Pinus sylvestris L. Scotch pine. En Burns, R.M.; Honkala, B.H.; eds. Silvics of North America. Volume 11. Conifers. Agric. Handb. 654. Washington D.C: U.S. Department of Agriculture, Forest Service. p. 489496.

Smith DM, BC Larson, JK Matthew, PMS Ashton. 1997. The practice of silviculture. New York, $9^{\text {th }}$ ed., John Wiley and Sons. 537 p.

Soil Survey Staff. 1999. Soil Taxonomy: a basic system of classification for making and interpreting soil surveys. $2^{\text {nd }}$ ed. Agriculture Handbook 436. Natural Resources Conservation Service, USDA, U.S. Gov. Printing Office. Washington D.C., Estados Unidos.

Soriano A. 1983. Deserts and semideserts of Patagonia. In West temperate deserts and semideserts. Amsterdam, Elsevier. p. 423-460.

Recibido: 04.03.04

Aceptado: 02.01.07 